\title{
Maternal And Neonatal Exposure To Risk Factors For Neonates With Moderate Or Severe Hypoxic Ischemic Encephalopathy: A Cross-Sectional Study
}

\author{
yiran wang ( $\nabla$ 872578928@qq.com ) \\ Zhengzhou Children's Hospital \\ shuying luo \\ Zhengzhou Children's Hospital \\ kaijuan wang \\ Zhengzhou University \\ yuwei hou \\ Zhengzhou Children's Hospital \\ hui yan \\ Zhengzhou Children's Hospital \\ yaodong zhang \\ Zhengzhou Children's Hospital
}

\section{Research Article}

Keywords: Hypoxic ischemic encephalopathy, Risk factors, cross-sectional study

Posted Date: February 28th, 2022

DOI: https://doi.org/10.21203/rs.3.rs-1371747/v1

License: (c) (1) This work is licensed under a Creative Commons Attribution 4.0 International License.

Read Full License 


\section{Abstract}

Background:To investigate the association between maternal and neonatal exposure to the relevant influencing factors and risk of moderate or severe hypoxic ischemic encephalopathy(HIE),and the possible interactions in the Chinese population.

Methods: A cross-sectional study comprising 228 neonates from Henan Children's Hospital during the fiveyear period 2015-2020 in China was conducted. All neonatal basic demographic information and clinical records were documented from the neonatal HIE database. Comparisons between mild HIE and moderate or severe HIE were conducted with the t-test or Wilcoxon rank-sum test for continuous variables and the Chi-square test for categorical variables. Unconditional multiple logistic regression models were used to generate the odds ratios(ORs) and $95 \%$ confidence intervals(Cls). In addition, we also used an additive model to test for possible biological interactions among the factors.

Results: Of the 228 neonates, the males had a statistically significantly higher frequency compared with the females between the two groups $(P=0.030)$. Trend analysis results found that with the decreased of the neonatal birth weight, the detection rates of moderate or severe HIE in males and females were gradually increased $\left(P_{\text {trend }} \llbracket 0.05\right)$. The detection of moderate or severe HIE in males and females increased with the decreased of neonatal gestational age at birth $\left(P_{\text {trend }} \otimes 0.05\right)$. However, no interaction was detected between neonatal birth weight and gestational age at birth based on the additive model, the Relative Excess Risk of Interaction and 95\% C/ was 3.856(-1.753,5.217). The adjusted multiple logistic regression model showed that low birth weight $\left(O R_{a d j} 1.965,95 \% \mathrm{Cl}: 1.086-4.127\right)$,premature

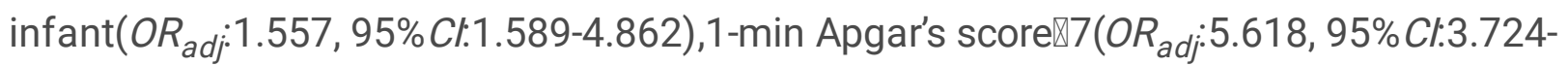
7.353), intrauterine distress $\left(O R_{a d j} 4.916,95 \%\right.$ Cl:3.431-7.398), amniotic fluid contamination ( $O R_{a d j}: 3.965$, 95\% Cl:2.153-5.782) significantly increased the risk of neonatal moderate or severe HIE.

Conclusion:Neonates with low birth weight, premature infant,1-min Apgar's score $\mathbb{7}$, intrauterine distress, amniotic fluid contamination are risk factors for moderate or severe HIE. Notably, we found no biological interaction between risk factors based on the additive model, these findings may help to inform prevention strategies, as this may effectively reduce the incidence of neonatal moderate or severe HIE.

\section{Introduction}

Neonatal Hypoxic ischemic encephalopathy (HIE) is a common disease caused by various factors in the whole perinatal period of neonates, which possibly causes neonatal hypoxia, reduced cerebral blood flow or suspension, thus resulting in varying degrees of brain damage ${ }^{[1-2]}$. Based on the neurological symptoms of the neonates, $\mathrm{HIE}$ is graded into three categories: mild, moderate and severe ${ }^{[3]}$. For neonates with mild HIE, outcomes are consistently positive,whereas neonates with moderate to severe HIE can suffer serious consequences, including death, cerebral palsy, epilepsy and cognitive disability ${ }^{[4-5]}$. Neonatal HIE accounts for $15.2 \%$ of mortality under the age of five, in addition, the mortality rate of neonatal moderate or severe HIE is high, and the rate of severe neurological disability in survivors is up to 
$55 \%-73 \%[6]$. Despite of significant improvements in neonatal care, HIE continues to exist in developed countries and a disproportionately high burden remains in low and middle income countries ${ }^{[7-11]}$. There are 15-18 million live births in China each year, and the incidence of neonatal HIE is estimated to be 3-6 per 1000 live births ${ }^{[12]}$.

Clinically, it is urgent need to identify the relevant risk factors of the neonatal moderate or severe HIE to take appropriate preventive and early intervention strategies before neonates occur. Previous epidemiological studies have demonstrated a number of medical and socioeconomic risk factors for neonatal moderate or severe $\mathrm{HIE}^{[13-16]}$, including maternal age, maternal obesity, gestational diabetes,viral infection, intrauterine growth retardation, neonatal mode of delivery, birth weight, gestational age, but these findings may somewhat inconsistent considering the changes in study designs, characteristics of the population, diagnostic criteria and outcome measures, meanwhile, several studies lacked analysis of the possible interactions among risk factors. Therefore, a cross-sectional study was undertaken in this study to identify the independent risk factors of neonatal moderate or severe HIE and also address the possible biological interactions used an additive model, in order to provide the necessary reference for early primary and secondary prevention in clinical.

\section{Methods}

\subsection{Study population}

This was a cross-sectional study comprising 228 neonates from Henan Children's Hospital during the fiveyear period 2015-2020 in China. All neonatal basic demographic information and clinical records were documented from the neonatal HIE database. The neonatal HIE database was set up in Henan Children's Hospital and known to be a province-wide, clinically-oriented computerized disease-specific database. We obtained information on the neonatal age at diagnosis, gender, residential region, birth weight and delivery mode from their birth certificate cards or vaccination cards and also collected related medical data from their medical records in the hospital. To ensure the reliability of the information, we routinely examined the items and logical errors in the database. When data was found to be inconsistent, we would list the numbers of the patients, record names, variable name and error categories to facilitate future checks and corrections.

For the current study, to focus on moderate and severe HIE, cases of HIE were defined by the following criteria:(a) all the cases were determined by the diagnostic criteria set by the Neonatal Group of the Pediatric Society of Chinese Medical Association; (b) all 228 neonates resided in the Neonatal Intensive Care Unit (NICU) of Henan Children's Hospital and had typical clinical manifestations (paroxysmal cyanosis, agitation, increased muscle tone, drowsiness, full anterior fontanelle, low muscle tone, binocular gaze, abnormal emission, screaming, convulsions, etc.), which were confirmed by head CT, magnetic resonance imaging (MRI) and other examinations;(c) the parents of the neonates were able ro cooperate closely with the investigation and the data were complete. According to clinical classification, neonatal HIE was divided into three categories: mild mainly manifested as agitation, normal or slightly active 
primitive reflex, no convulsion; moderate (mainly with inhibition symptoms, manifested as drowsiness, decreased muscle tone, weakened primitive reflex, partially with increased intracranial pressure and convulsion), severe (mainly with coma, the manifestations are loss of muscle tone of limbs, no spontaneous movement, disappearance of primitive reflex, most of them have increased cranial pressure and convulsion). The exclusion criteria for neonates included patients with incomplete clinical data and with congenital cardiopulmonary diseases, genetic or metabolic diseases.

\subsection{Ascertainment of variables}

Background data and exposure to considered potential risk factors were extracted from the neonatal HIE database.The possible risk factors studied included neonatal birth weight, gestational age at birth, parity(one, two,three or more), feeding pattern(breastfeeding,artificial feeding or mixed feeding),delivery mode(natural delivery or cesarean section),1-min Apgar's score ( $\geq 7$ or $\unrhd 7$ ), 5 -min Apgar's score( $\geq 7$ or $\otimes 7$ ), preterm birth, intrauterine infection, intrauterine distress, amniotic fluid contamination, amniotic fluid anomaly and abnormal labor stage. In this study, neonatal birth weight was categorized as low birth

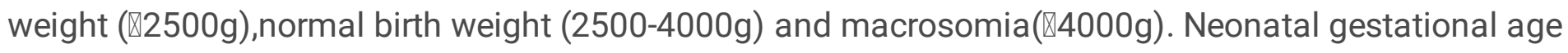

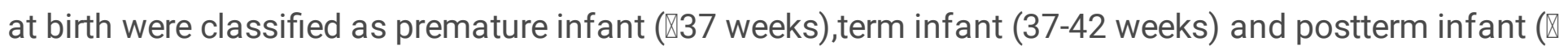
42 weeks). Intrauterine distress was referred to neonatal heart less than 120 beats/min or more than 160 beats/min. Amniotic fluid anomaly was defined as the amniotic fluid volume $\geq 2000 \mathrm{ml}$ or $\leq 300 \mathrm{ml}$, and the amniotic fluid dark area $\$ 7 \mathrm{~cm}$ or $\leq 2 \mathrm{~cm}$ by B-ultrasound examination. Abnormal labor stage was referred to the total labor course $\leq 3 \mathrm{~h}$ or $\geq 24 \mathrm{~h}$, the second labor stage $\geq 2 \mathrm{~h}$ for primipara and $\geq 1 \mathrm{~h}$ for parturient. Neonatal preterm birth, intrauterine infection, intrauterine distress, amniotic fluid contamination, amniotic fluid anomaly and abnormal labor stage were all classified as a dichotomous variable "Yes" or "No".

\subsection{Statistical analysis}

Maternal and neonatal baseline descriptive characteristics with continuous variables and categorical variable were all presented as mean \pm standard deviation value and frequencies and percentages respectively. Comparisons between different levels neonatal HIE were conducted with the t-test or Wilcoxon rank-sum test for continuous variables and the Chi-square test for categorical variables. Considering that there still were nonmatching variables among different levels neonatal HIE apart from age, gender, and residence region, we carried out unconditional multiple logistic regression models to estimate the independent risk factors of interest for predicting neonatal moderate or severe HIE. Collinearity between potential confounding variables was examined using Spearman rank-order correlation for continuous variables or Chi-square test for category variables. All variables identified as statistically significant $(P<0.05)$ predictors in the univariate analysis were included as candidate predictors in the multivariate model. Odds ratios(ORs) of mild HIE vs. moderate or severe HIE were reported along with the corresponding $95 \%$ confidence intervals (Cls).First, we estimated the unadjusted ORs by each maternal and neonatal characteristic in model 1. Thereafter, we adjusted ORs for the different maternal and neonatal characteristics for each other, based on associations in present data and in previous reports in model 2. 
To further explore the characteristics of 228 males and females neonatal HIE in different group, we conducted trend test analysis stratified by the neonatal birth weight and gestational age at birth. In addition, we also used an additive model to test for possible biological interactions between neonatal birth weight and gestational age at birth. OR was calculated for each category after adjustment for covariates and Relative Excess Risk of Interaction (RERI) was calculated based on the additive model. All statistical analyses were conducted with IBM software SPSS (version 21, Chicago, IL, USA). All statistical tests were two-tailed and considered to be statistically significant at $P$ value less than 0.05 .

\section{Results}

A total of 228 neonates recorded in the Henan Children's Hospital Patient Register as diagnosed with neonatal HIE were included in this cross-sectional study. Of these, 21(9.2\%) neonates were diagnosed with mild HIE, 207(90.8\%) neonates were diagnosed with moderate or severe HIE. The demographic characteristics of mild HIE and moderate or severe HIE were summarized in Table 1. The males had a statistically significantly higher frequency compared with the females between the two groups $(P=0.030)$. However, there were no difference between the groups with respect to the residential region $(P=0.423)$. In the population, $171(75.0 \%)$ neonatal birth weight were categorized as low birth weight $(\mathbb{2} 2500 \mathrm{~g}), 33(14.5 \%)$ as normal birth weight (2500-4000 g) and $24(10.5 \%)$ as macrosomia( $(4000 \mathrm{~g}) .157(67.5 \%)$ neonatal gestational age at birth were categorized as premature infant ( $₫ 37$ weeks),43(18.9\%) as term infant (3742 weeks) and $31(13.6 \%)$ as postterm infant ( $\$ 42$ weeks). There were statistically significantly difference between the two groups regarding neonatal birth weight and gestational age at birth(Pख0.05). Meanwhile, all other considered characteristics tested with significant difference between mild HIE and moderate or severe HIE(all $P \otimes 0.05)$.

The detection and trend analysis results of mild HIE and moderate or severe HIE among neonates with different birth weight and different gestational age at birth were presented in Table 2. We found a trend association between different levels neonatal HIE and birth weight, with the decreased of the neonatal birth weight, the detection rates of mild HIE and moderate or severe HIE in males and females were gradually increased (all $P_{\text {trend }} \mathbb{\otimes 0 . 0 5}$ ). The detection rates of different levels HIE in males and females were

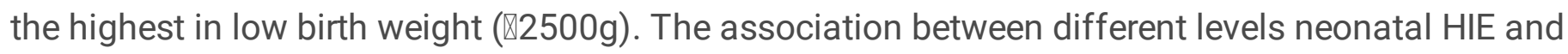
gestational age at birth also showed a tendency, with the decreased of the neonatal gestational age at birth, the detection rates of mild HIE and moderate or severe HIE in males and females were gradually increased (all $P_{\text {trend }} \mathbb{\nabla 0 . 0 5}$ ). The detection rates of different levels HIE in males and females were the highest in premature infant ( $₫ 37$ weeks).

Multivariate logistic regression analysis of influencing neonatal moderate or severe HIE was shown in

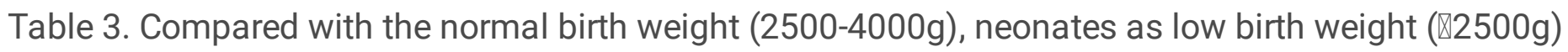
(adjusted OR:1.965, 95\% Cl:1.086-4.127)was independent risk factor of moderate or severe HIE after adjusting for potential confounders in model 2. Also, neonatal gestational age at birth exposed to premature infant ( $₫ 37$ weeks) (adjusted OR:1.557, 95\% Cl:1.589-4.862) increased the risk of moderate or severe HIE compared with term infant (37-42 weeks). The RER/ and $95 \% \mathrm{Cl}$ in neonatal HIE was 
3.856(-1.753,5.217) and indicated that no interaction was detected between neonatal birth weight and gestational age at birth based on the additive model. In addition, the results revealed that neonatal 1-min Apgar's score 7 , intrauterine distress, amniotic fluid contamination were significantly associated with an increased risk of moderate or severe HIE.

\section{Discussion}

Moderate or severe HIE is one of the most common diseases in neonatal period.Therefore, understanding the onset and related risk factors affecting the neonatal moderate or severe $\mathrm{HIE}$, can help clinicians take preventive measures and improve the prognosis of neonates thus reducing the mortality and disability rate in time. The development of moderate or severe HIE is caused by the interaction of multiple factors throughout the neonatal period. In this cross-sectional study, we compared the differences among various factors of neonates with moderate and severe HIE, and the results showed that five factors was associated with higher risk of neonatal moderate or severe HIE after adjusting for other variables. The adjusted logistic regression analysis showed significant associations between neonatal moderate or severe HIE and low birth weight, premature infant,1-min Apgar's score『7, intrauterine distress and amniotic fluid contamination. No biological interaction was detected between neonatal birth weight and gestational age at birth based on the additive model.

In the neonatal factors, our study showed that low birth weight was associated with an increased risk of moderate or severe HIE(adjusted OR:1.965, 95\% Cl:1.086-4.127). The trend analysis results also found that with the decreased of the neonatal birth weight, the detection rates of moderate or severe HIE in males and females were gradually increased (all $P_{\text {trend }} \otimes 0.05$ ), and the detection rates of moderate or severe HIE in males and females were the highest in low birth weight. The relationship between neonatal premature infant and moderate or severe HIE also showed the same results. Our finding was consistent with the previous literature, in a retrospective study of 17706 newborns, Futrakul et al. revealed that gestational age were significant risk factors of $\mathrm{HIE}^{[17]}$. Neonatal low birth weight and premature infant were always accompanied by imperfect organ development, especially immature lung development, which was easily lead to the occurrence of moderate or severe HIE. However, no biological interaction between neonatal birth weight and gestational age at birth based on the additive model was found in this study, that meant when multiple risk factors interact with moderate or severe HIE, the effect was equivalent to the sum of the independent effects of the two factors.

It has been suggested that the Apgar's score is a feasible and practical indicator to evaluate the degree of neonatal moderate or severe HIE, especially the 1-min Apgar's score is still a valid and rapid index to assess the effectiveness of resuscitative efforts and the presence and degree of respiratory depression in neonates ${ }^{[18]}$. Our study indicated that there was a statistically significant relationship between 1-min

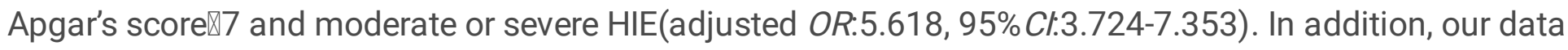
reported that intrauterine distress was also associated with a significant increase in risk of neonatal moderate or severe HIE (adjusted OR:4.916, 95\% Cl:3.431-7.398). The present study supported the previous report by Wang J ${ }^{[19]}$, that Apgar's score (OR:5.648, 95\% Cl:3.704-9.241) and intrauterine 
distress(OR:3.662, 95\% Cl:2.085-4.736) were independent risk factors for HIE. Several studies have demonstrated that the continuous lower Apgar's score indicated the higher degree of neonatal moderate or severe HIE, which can caused hypoxia and acidosis, redistributing blood, significant abnormalities of residual alkali,blood glucose and failure of brain energy metabolism, resulting in brain edema and cell damage, or death in severe neonates ${ }^{[20-21]}$. Hypoxia caused by low Apgar's score is also a continuation result of intrauterine distress in utero mostly. Intrauterine distress is the manifestation of acute or chronic fetal hypoxia. Long-term hypoxia can cause acidosis, inhibit respiratory center and lead to neonatal moderate or severe HIE. Therefore, these results remind that it is necessary to carry out the 1-min Apgar's score and disease degree assessment in time to help clinicians to take targeted treatment measures and strengthen the monitoring of the whole labor process, thus avoid the occurrence of asphyxia and reduce the brain damage caused by asphyxia in neonatal moderate or severe HIE.

Amniotic fluid contamination was also a risk factor in this study and has received a lot of attention. Our study concluded that after adjusting for potential confounders in multiple logistic regression models, neonates with amniotic fluid contamination were 3.965 times more likely to develop moderate or severe HIE than those without amniotic fluid contamination(adjusted $O R: 3.965,95 \% \mathrm{Cl}: 2.153-5.782$ ). Our finding was consistent with the previous literature, Wang $\mathrm{J}$ et al. carried out a retrospective case-control study in southern China, in which they found that $O R$ for neonatal HIE associated with amniotic fluid contamination was $4.527(95 \% \mathrm{Cl}: 2.704-5.483)$ (using 306 cases, 306 controls) ${ }^{[19]}$. Some studies have suggested that neonate exposed to amniotic fluid contamination is an important factor leading to intrauterine distress, and there is likely to be a positive association between amniotic fluid contamination and neonatal moderate or severe $\mathrm{HIE}^{[22-23]}$. The higher degree of amniotic fluid contamination, the higher probability of neonates with moderate or severe HIE. Neonates with intrauterine hypoxia, hyperperistalsis, anal sphincter relaxation, can make meconium discharge into the amniotic fluid. Currently amniotic fluid contamination is a clinical manifestation of neonatal intrauterine hypoxia, the incidence of neonatal HIE increases when amniotic fluid contamination occurs. Therefore, prenatal examination should be used to monitor whether the amniotic fluid is contaminated. Once the neonate is in distress, correct countermeasures should be made to end the delivery with appropriate methods timely.

The main strengthens of the present study are the enrolled population of neonates came from a single hospital institution and adjustment for various covariates in the analysis, thus minimizing differences in outcome assessment and limiting information bias. But several limitations of the study should be noted. Firstly, our study mainly focused on the neonatal moderate or severe HIE, such restrictions lead to a reduction in the sample size of our study, which may have caused some variables with lack of significance. Secondly, given that the study was conducted in a cross-sectional design, the causal pathways underlying the observed relationships could hardly be verified. Further cohort studies are needed if permitted. The selected controls in this study were recruited among neonates who were diagnosed with mild HIE, some factors which limited to data collected from patients' medical records could still differ between cases and controls, leading to unrecognized selection bias. It is very hard to balance all these baseline factors in the process of recruiting cases and controls. Thirdly,utilizing 
diagnosis codes to identify cases which may caused small number of cases cannot be screened out with this method, some of the risk estimates were based on small numbers and some might be statistically significant simply by chance alone, this may have impacted our ability to establish an association between neonatal moderate or severe HIE and other factors. Our finding need to be replicated by other investigations in the future with lager sample sizes.

\section{Conclusions}

In conclusion, findings in our study are in line with the previous literatures, which show that neonates with low birth weight, premature infant,1-min Apgar's score\7, intrauterine distress, amniotic fluid contamination are risk factors for moderate or severe HIE. Notably, we found no biological interaction between risk factors based on the additive model. It is important to pay close attention to these risk factors, which may help to inform prevention and early detection strategies, as this may succeed in minimizing the incidence of neonatal moderate or severe HIE.

\section{List Of Abbreviations}

\begin{tabular}{|ll|}
\hline Odds Ratios & ORs \\
\hline Confidence Intervals & Cls \\
\hline Relative Excess Risk of Interaction & RERI \\
\hline
\end{tabular}

\section{Declarations}

\section{Ethics approval and consent to participate}

Not applicable

\section{Consent for publication}

Not applicable

\section{Availability of data and materials}

The datasets used and/or analysed during the current study are available from the corresponding author on reasonable request.

\section{Competing interests}

The authors declare that they have no competing interests.

\section{Funding}

No funding was received for this research. 


\section{Authors' contributions}

$Y$ W wrote the first draft and final article versions of this paper. $Y \mathrm{~W}$ and $\mathrm{Y} Z$ contributed to the conception and design of the study. S L, Y H and $\mathrm{H} \mathrm{Y} \mathrm{participated} \mathrm{in} \mathrm{investigations} \mathrm{and} \mathrm{data} \mathrm{collection.} \mathrm{K} W$ was responsible for data cleaning and analysis. All authors read and approved the final manuscript.

\section{Acknowledgements}

Not applicable

\section{References}

[1] Malmqvist O, Ohlin A, Agren J, et al.Seizures in newborn infants without hypoxic ischemic encephalopathy-antenatal and labor-related risk factors: a case-control study.J Matern Fetal Neonatal Med.2020;33(5):799-05.

[2] Scheidegger S, Held U, Grass B, et al.Association of perinatal risk factors with neurological outcome in neonates with hypoxic ischemic encephalopathy.J Matern Fetal Neonatal Med.2021;34(7):1-7.

[3] Sarnat HB, Sarnat MS. Neonatal encephalopathy following fetal distress. A clinical and electroencephalographic study. Arch Neurol. 1976;33:696-05.

[4] Kurinczuk JJ, White-Koning M, Badawi N. Epidemiology of neonatal encephalopathy and hypoxicischaemic encephalopathy. Early Hum Dev. 2010;86:329-38.

[5] Jacobs SE, Berg M, Hunt R, et al. Cooling for newborns with hypoxic ischaemic encephalopathy. Cochrane Database Syst Rev. 2013;1:1-23.

[6] Song P, Theodoratou E, Li X, et al. Causes of death in children younger than five years in China in 2015: an updated analysis. J Glob Health. 2016;6(2):1-13.

[7] Wang Z, Zhang P, Zhou WH, et al.Neonatal hypoxic-ischemic encephalopathy diagnosis and treatment: a National Survey in China. BMC Pediatrics.2021;21(261):1-9.

[8]Vannucci RC. Hypoxic-ischemic encephalopathy. Am J Perinatol. 2000;17(3):113-20.

[9] Liu L, Oza S, Hogan D, Chu Y, et al. Global, regional, and national causes of under-5 mortality in 200015: an updated systematic analysis with implications for the sustainable development goals. Lancet. 2016;388(10063):3027-35.

[10] Kurinczuk JJ, White-Koning M, Badawi N. Epidemiology of neonatal encephalopathy and hypoxicischemic encephalopathy. Early Hum Dev. 2010;86(6):329-38. 
[11] Pauliah S, Shankaran S, Wade A, et al. Therapeutic hypothermia for neonatal encephalopathy in lowand middle-income countries: a systematic review and meta-analysis. PLOS One. 2013;8(3):1-8.

[12] Shao XM, Ye HM, Qiu XS. Practice of neonatology. Version 4. Beijing:People's Medical Publishing House. 2011;p. 699-06.

[13] Lundgren C, Brudin L, Wanby AS, et al. Ante- and intrapartum risk factors for neonatal hypoxic ischemic encephalopathy. J Matern Fetal Neonatal Med. 2018;31(12):1-7.

[14] Torbenson VE, Tolcher MC, Nesbitt KM, et. al.Intrapartum factors associated with neonatal hypoxic ischemic encephalopathy: a case-controlled study. BMC Pregnancy and Childbirth. 2017;17(1):1-7.

[15] Rossi AC, Prefumo F. Antepartum and intrapartum risk factors for neonatal hypoxic-ischemic encephalopathy: a systematic review with meta-analysis. Curr Opin Obstet Gynecol. 2019;31:411-6.

[16] Parker SJ, Kuzniewicz M, Niki H, et al. Antenatal and intrapartum risk factors for hypoxic-ischemic encephalopathy in a US birth cohort. J Pediatr. 2018;203:163-9.

[17] Futraful S, Praisuwanna P, Thaitumyanon P.Risk factors for hypoxic-ischemic encephalopathy in asphyxiated newborn infants.J Med Assoc Thai.2006;89(3): 322-8.

[18] Casey BM, Mclntire DD, Leveno KJ. The continuing value of the Apgar score for the assessment of newborn infants. N Engl J Med. 2001; 344(7):467-71.

[19] Wang J,Tao E, Mo M, et al.Perinatal Risk factors influencing neonatal hypoxic ischemic encephalopathy in southern China:a case-control study. Am J Perinatol.2020;3:1-5.

[20] Wood S, Crawford S, Hicks M, et al. Hospital-related,maternal, and fetal risk factors for neonatal asphyxia and moderate or severe hypoxic-ischemic encephalopathy: a cross-sectional study. J Matern Fetal Neonatal Med.2021;34(9):1-7.

[21] Mwaniki MK, Atieno M, Lawn JE, et al. Long-term neurodevelopmental outcomes after intrauterine and neonatal insults: a systematic review. Lancet.2012;379 (9814): 445-52.

[22] Chen HM, Gao LX, Wang JJ,et al.The correlation between AGT gene polymorphism and neonatal hypoxic-ischemic encephalopathy (HIE). Eur Rev Med Pharmacol Sci. 2019;23(5):2194-9.

[23] Kujala S, Waiswa P, Kadobera D, et al. Trends and risk of risk factors of stillbirths and neonatal deaths in Eastern Uganda(1982-2011):a cross-sectional, population-based study.Trop Med Int Health.2017;22(1):63-73.

\section{Tables}


Table 1. Maternal and neonatal demographic characteristics with different levels neonatal hypoxic ischemic encephalopathy 
Variable

Total

mild HIE

$\mathrm{n}(\%) \quad \mathrm{n}(\%) \quad \mathrm{n}(\%)$

Age at diagnosis(days)

$13.9 \pm 5.5$

$14.1 \pm 5.7$

$13.7 \pm 5.2$

0.061

Gender

Male
Female
Residential region

Urban

90(39.5) 10(47.6) 80(38.6)

0.423

Rural

138(60.5) 11(52.4) 127(61.4)

Birth weight(g)

\begin{tabular}{|c|}
\hline$\otimes 2500$ \\
\hline $2500-4000$ \\
\hline$₫ 4000$ \\
\hline
\end{tabular}

\37

171(75.0) 11(52.4) 160(77.3)

$33(14.5) \quad 6(28.6) \quad 27(13.0)$

$24(10.5) \quad 4(19.0) \quad 20(9.7)$

37-42

\begin{tabular}{|lll}
\hline $154(67.5)$ & $9(42.9)$ & $145(70.0)$ \\
\hline $43(18.9)$ & $7(33.3)$ & $36(17.4)$ \\
\hline $31(13.6)$ & $5(23.8)$ & $26(12.6)$
\end{tabular}

$₫ 42$

\section{Parity}

\begin{tabular}{|c|c|c|c|c|}
\hline 1 & $134(58.7)$ & $8(38.1)$ & 126(60.9) & \multirow[t]{3}{*}{0.034} \\
\hline 2 & $64(28.1)$ & $11(52.4)$ & $53(25.6)$ & \\
\hline$\geq 3$ & $30(13.2)$ & $2(9.5)$ & 28(13.5) & \\
\hline \multicolumn{5}{|l|}{ Feeding pattern } \\
\hline Breastfeeding & $135(59.2)$ & $9(42.9)$ & 126(60.9) & \multirow[t]{3}{*}{0.035} \\
\hline Artificial feeding & $56(24.6)$ & $10(47.6)$ & $46(22.2)$ & \\
\hline Mixed feeding & $37(16.2)$ & $2(9.5)$ & $35(16.9)$ & \\
\hline \multicolumn{5}{|l|}{ Delivery mode } \\
\hline Natural delivery & 134(58.8) & $8(38.1)$ & 126(60.9) & \multirow[t]{2}{*}{0.043} \\
\hline Cesarean section & $94(41.2)$ & 13(61.9) & 81(39.1) & \\
\hline
\end{tabular}




\begin{tabular}{lllll}
$\geq 7$ & $137(60.1)$ & $7(33.3)$ & $130(62.8)$ & 0.009 \\
\cline { 1 - 3 } & $91(39.9)$ & $14(66.7)$ & $77(37.2)$ & \\
\hline
\end{tabular}

5-min Apgar's score

\begin{tabular}{|c|c|c|c|c|}
\hline$\geq 7$ & $116(50.9)$ & $15(71.4)$ & $101(48.8)$ & 0.048 \\
\hline$\otimes 7$ & $112(49.1)$ & $6(28.6)$ & $106(51.2)$ & \\
\hline
\end{tabular}

Preterm birth

\begin{tabular}{|c|c|c|c|c|}
\hline Yes & $172(75.4)$ & $11(52.4)$ & 161(77.8) & \multirow[t]{2}{*}{0.010} \\
\hline No & $56(24.6)$ & $10(47.6)$ & $46(22.2)$ & \\
\hline \multicolumn{5}{|c|}{ Intrauterine infection } \\
\hline Yes & $98(43.0)$ & 15(71.4) & $83(40.1)$ & \multirow[t]{2}{*}{0.006} \\
\hline No & $130(57.0)$ & $6(28.6)$ & 124(59.9) & \\
\hline \multicolumn{5}{|c|}{ Intrauterine distress } \\
\hline Yes & $119(52.2)$ & $16(76.2)$ & $103(49.8)$ & \multirow[t]{2}{*}{0.021} \\
\hline No & $109(47.8)$ & $5(23.8)$ & 104(50.2) & \\
\hline \multicolumn{5}{|c|}{ Amniotic fluid contamination } \\
\hline Yes & $122(53.5)$ & $16(76.2)$ & 106(51.2) & \multirow[t]{2}{*}{0.029} \\
\hline No & $106(46.5)$ & $5(23.8)$ & 101(48.8) & \\
\hline \multicolumn{5}{|c|}{ Amniotic fluid anomaly } \\
\hline Yes & $130(57.0)$ & 17(81.0) & $113(54.6)$ & \multirow[t]{2}{*}{0.020} \\
\hline No & $98(43.0)$ & $4(19.0)$ & $94(45.4)$ & \\
\hline \multicolumn{5}{|c|}{ Abnormal labor stage } \\
\hline Yes & $172(75.4)$ & $12(57.1)$ & 160(77.3) & \multirow[t]{2}{*}{0.041} \\
\hline No & $56(24.6)$ & $9(42.9)$ & $47(22.7)$ & \\
\hline
\end{tabular}

Table 2. Detection and trend test analysis of different levels HIE among neonates with different groups 


\begin{tabular}{|c|c|c|c|c|c|c|}
\hline \multirow[t]{2}{*}{ Group } & \multicolumn{3}{|l|}{ Male } & \multicolumn{3}{|c|}{ Female } \\
\hline & Total & $\begin{array}{l}\text { mild } \\
\mathrm{HIE}\end{array}$ & $\begin{array}{l}\text { moderate or } \\
\text { severe HIE }\end{array}$ & Total & $\begin{array}{l}\operatorname{mild}_{\mathrm{HIE}} \\
\text { ile }\end{array}$ & $\begin{array}{l}\text { moderate or } \\
\text { severe HIE }\end{array}$ \\
\hline \multicolumn{7}{|c|}{ Birth weight(g) } \\
\hline$\varangle 2500$ & 135 & $6(50.0)$ & 129(79.6) & 36 & $5(55.6)$ & $31(68.9)$ \\
\hline $2500-4000$ & 23 & $5(41.7)$ & 18(11.1) & 12 & $3(33.3)$ & $9(20.0)$ \\
\hline$\nabla 4000$ & 16 & $1(8.3)$ & 15(9.3) & 6 & $1(11.1)$ & $5(11.1)$ \\
\hline$P_{\text {trend* }}$ & & 0.016 & 0.028 & & 0.021 & 0.039 \\
\hline \multicolumn{7}{|c|}{$\begin{array}{l}\text { Gestational age at } \\
\text { birth(weeks) }\end{array}$} \\
\hline$\varangle 37$ & 115 & $5(41.7)$ & 110(67.9) & 39 & $6(66.7)$ & $33(73.3)$ \\
\hline $37-42$ & 31 & $4(33.3)$ & $27(16.7)$ & 13 & $2(22.2)$ & $11(24.4)$ \\
\hline$\$ 42$ & 28 & $3(25.0)$ & $25(15.4)$ & 2 & $1(11.1)$ & $1(2.3)$ \\
\hline$P_{\text {trend* }}$ & & 0.023 & 0.035 & & 0.037 & 0.042 \\
\hline
\end{tabular}

Table 3. Independent risk factors for neonatal moderate or severe HIE based on logistic regression analysis

${ }^{a}$ Model 1: the unadjusted ORs by each maternal and neonatal characteristic; ${ }^{b}$ Model 2: adjusted ORs for the different maternal and neonatal characteristics for each other. ${ }^{\star} 1.00$ (ref) meant the reference group. 
Variable

Model $1^{\mathrm{a}}$

Unadjusted

$\mathrm{OR}(95 \% \mathrm{Cl})$
$P$ -

value
Model $2^{b}$

Adjusted OR(95\% Cl) $\quad P$ -

value

Birth weight(g)

$\$ 2500$

1.701(1.153 3.082) $\quad 0.019$

1.965(1.086 4.127)

0.036

2500-4000

1.00 (ref)*

1.00 (ref)

$₫ 4000$

2.879(0.234 4.328)

Gestational age at birth(weeks)

\begin{tabular}{|c|c|c|c|c|}
\hline$\varangle 37$ & $1.152(1.067 \sim 2.985)$ & \multirow[t]{3}{*}{0.027} & $1.557(1.589 \sim 4.862)$ & \multirow[t]{3}{*}{0.041} \\
\hline $37-42$ & 1.00 (ref) & & 1.00 (ref) & \\
\hline$₫ 42$ & $2.983(0.719 \sim 5.106)$ & & $3.027(0.165 \sim 6.017)$ & \\
\hline
\end{tabular}

1-min Apgar's score

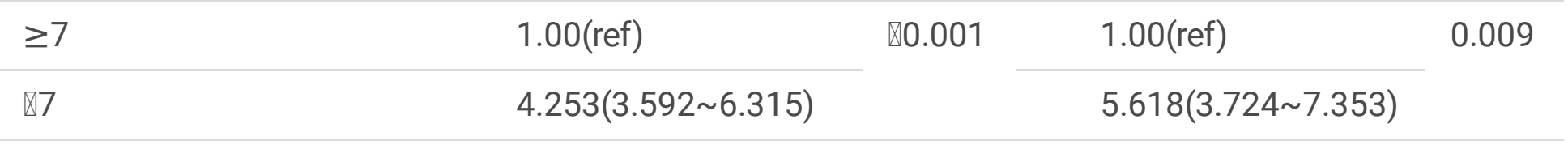

Intrauterine distress

\begin{tabular}{|c|c|c|c|c|}
\hline Yes & $5.921(3.565 \sim 6.828)$ & \multirow[t]{2}{*}{0.002} & $4.916(3.431 \sim 7.398)$ & \multirow[t]{2}{*}{0.011} \\
\hline No & 1.00 (ref) & & 1.00 (ref) & \\
\hline
\end{tabular}

Amniotic fluid contamination

\begin{tabular}{|c|c|c|c|c|}
\hline Yes & $2.775(1.986 \sim 4.357)$ & $\otimes 0.001$ & $3.965(2.153 \sim 5.782)$ & 0.025 \\
\hline No & 1.00 (ref) & & 1.00 (ref) & \\
\hline
\end{tabular}

